André Guillerme, La naissance de l'industrie à Paris, entre sueurs et vapeurs 1780-1830

Seyssel, Champ Vallon, 2007, 439 pages.

Michel Cotte

(2) OpenEdition

Journals

Édition électronique

URL : http://journals.openedition.org/dht/1073

DOI : $10.4000 /$ dht. 1073

ISSN : $1775-4194$

Éditeur :

Centre d'histoire des techniques et de l'environnement du Cnam (CDHTE-Cnam), Société des élèves du CDHTE-Cnam

Édition imprimée

Date de publication : 1 juin 2008

Pagination : 228-230

ISBN : 978-2-95-30779-1-9

ISSN : 0417-8726

Référence électronique

Michel Cotte, «André Guillerme, La naissance de l'industrie à Paris, entre sueurs et vapeurs 1780-1830», Documents pour l'histoire des techniques [En ligne], 15 | $7^{\text {er }}$ semestre 2008, mis en ligne le 22 octobre 2010, consulté le 23 septembre 2020. URL : http://journals.openedition.org/dht/1073 ; DOI : https:// doi.org/10.4000/dht.1073

Ce document a été généré automatiquement le 23 septembre 2020.

(ㄷ) Tous droits réservés 


\title{
André Guillerme, La naissance de l'industrie à Paris, entre sueurs et vapeurs 1780-1830
}

Seyssel, Champ Vallon, 2007, 439 pages.

\author{
Michel Cotte
}

\section{RÉFÉRENCE}

André Guillerme, La naissance de l'industrie à Paris, entre sueurs et vapeurs 1780-1830, Seyssel, Champ Vallon, 2007, 439 pages.

1 La dernière livraison d'André Guillerme est dans la pleine continuité de ses précédents travaux les plus importants: Les temps de l'eau (1983), Bâtir la ville (1995) et, en collaboration, Le sol urbain (2001). Il poursuit sa quête de la réalité matérielle des civilisations urbaines. Il approfondit et fouille les substrats de la production matérielle des villes. Il en explore les conditions pratiques, observe les transformations professionnelles, sonde le savoir-faire des métiers, examine les progrès des sciences pratiques, enregistre les conditions de travail et les maladies professionnelles. Il évalue les quantités de matières premières, les produits finis, les volumes, les marchés, les prix, les odeurs, les sueurs, les pollutions, les vapeurs... Il fait œuvre d'écologie humaine historique.

2 Son sujet est cette fois la question des origines de l'industrie à Paris, durant une période résolument à cheval sur les XVIII ${ }^{\mathrm{e}}$ et $\mathrm{XIX}^{\mathrm{e}}$ siècles. Il nous a déjà habitués à une telle périodisation, bien peu classique mais tellement riche et dont il confirme être l'un des grands spécialistes. Traditionnelle, en France, la césure entre époques moderne et contemporaine tend à produire des spécialistes universitaires d'avant la Révolution de 1789 ou d'après, et la capacité à embrasser solidement les années qu'il explore est bien rare. Et pourtant, il s'agit de deux générations seulement, pas plus! Nombre de Parisiens nés dans les années 1760-1770 ont pu être les témoins directs de la totalité des 
faits qu'il nous propose d'examiner. La période est importante, car elle coïncide grosso modo avec la première phase de la révolution industrielle anglaise, celle du machinisme cotonnier, du charbon, des canaux, de la fonte au coke et des machines à vapeur. C'est une sorte de tableau en creux de cette révolution qui nous est proposé par André Guillerme : Londres est presque toujours là, entre les lignes, comme le véritable contrepoint de son étude parisienne. Le mimétisme devrait être la règle car tout ce beau monde des métiers et de la technique se connaît, malgré la Manche, malgré les guerres et les soit disants secrets industriels. Tout est comparable, mais rien n'est pareil! Et si deux modèles complètement différents avaient généré deux voies bien distinctes d'accès à des productions industrielles en milieu urbain, celles-ci ne convergeant que sur le tard et sans doute moins qu'on ne l'a dit? Et si l'historiographie classique avait complètement sous-estimé la dynamique artisanale et de micro entreprises familiales que l'on assimilerait aujourd'hui à des secteurs économiques informels? Si l'on veut comprendre ce qui se passe vraiment dans le domaine de la civilisation matérielle, il ne faut pas craindre d'embrasser cette période cruciale, d'en transcender les événements politiques sans les oublier pour autant, de retrousser ses manches et de descendre avec l'auteur dans les ateliers parisiens pour aller voir et sentir ce qui s'y passe vraiment.

3 André Guillerme s'est donné les moyens de son projet en compilant principalement deux types de données : d'un côté les fascicules des entreprises, les manuels, les revues et les rapports techniques sur "les arts et métiers", déjà très nombreux à cette période, de l'autre les rapports de l'administration également fort nombreux, de police et de salubrité publique notamment. L'un des résultats de cette enquête méticuleuse est une réévaluation très importante de la population parisienne réellement en activité manuelle et technique dans la capitale, par comparaison aux statistiques d'ensemble, généralement à la base de l'historiographie classique qui, du coup, sous-estime fortement la dimension industrielle de Paris à cette époque. La capitale française n'est pas moins développée que Londres, ou en retard, elle est différente! À la révolution industrielle lourde, mécanique et charbonneuse des uns s'opposerait une révolution industrieuse artisanale, chimique, biologique, légère et obnubilée par le blanc pour les autres..

Dans sa première partie, Le biotope, André Guillerme nous donne à voir tout ce dont Paris tire d'elle-même, de son substrat minéral et organique, avec talent et opiniâtreté. Il s'agit d'une foule de petits métiers utilisant ses eaux, aussi indispensables que fragiles, vite chargées en sels et en acidité par les nombreuses activités humaines. La récupération de ses sécrétions minérales lui fournit la première mine de salpêtre d'Europe, véritable nerf de la guerre. Paris utilise aussi son ossuaire gigantesque, probablement unique, qu'elle transforme en une masse considérable de produits, dont on imagine mal l'importance aujourd'hui. Les applications de l'os solide sont multitudes : boutons, cornets, peignes, tabletières, etc. ; la gélatine pour l'alimentation humaine, la colle; ensuite l'os comme engrais, une fois calciné comme noir animal dont les applications sont de plus en plus variées. À chaque fois, une plongée dans les métiers s'effectue, les réseaux de clients et de fournisseurs, l'évolution des pratiques et des marchés, les nouveaux acteurs, le rôle des inventions, l'implication des professions savantes dans les activités professionnelles: pharmaciens, chimistes, mécaniciens, etc. Nous voilà rendus aux activités de récupération, qui font vivre une masse de gens, des plus pauvres à de véritables hommes d'affaires: la cendre est une matière première précieusement collectée, les chiffons pour le papier, le carton; les métiers de 
l'équarrissage et de la vidange sont aussi à l'origine de matières premières indispensables à l'activité urbaine. À chaque fois, André Guillerme essaie de cerner le nombre d'acteurs et les évolutions sur la période, les quantités matérielles en jeu. Ces données sont souvent saisissantes, montrant des croissances impressionnantes et des montées en puissances parfois fulgurantes.

5 La seconde partie, La révolution biochimique, décrit le corps à corps de la ville avec les animaux et les matières végétales nécessaires à son alimentation, mais à bien d'autres choses aussi. C'est une véritable "génétique des arts» qui se produit alors et qui consiste à utiliser de manière de plus en plus raffinée et renouvelée ces nombreuses matières organiques: boyaux traités aux innombrables applications, colles, huiles, chandelles dont la production se renouvelle par l'isolement de la stéarine, féculerie et amidonnerie, etc. Rien n'est moins stable que ces différents métiers; il y a par exemple de nombreuses façons de faire de la colle qui entrent en concurrence. Marchés et techniques se renouvellent rapidement. De nombreux composés chimiques sont également extraits de ces ressources, alcools, colorants, etc. Les très nombreux métiers du cuir sont encore une spécificité parisienne, en prise directe avec un marché exigeant et nombreux, comme la plupart des productions rencontrées. La chapellerie, basée sur le feutre de poil, est l'une des premières industrialisations parisiennes véritablement accomplie, à la fois basée sur un « secret » de traitement des poils et sur la participation à ce formidablement mouvement mondial de collecte des peaux. L'un des ressorts de la réussite du modèle économique parisien, vite tourné vers le luxe et la diversification des productions, est bien là, déjà souligné par un auteur comme Louis Bergeron.

6 L'industrie parisienne pure et dure, parce qu'il faut bien y venir, est d'abord chimique et minérale, très proche de sa dimension scientifique et souvent animée par des savants. Les chimistes parisiens sont nombreux, compétents et généralement entreprenants. Ils produisent des acides en tout genre, sulfurique, chlorhydrique, nitrique, pyroligneux; ensuite de la soude, des sels minéraux; tous produits indispensables à tant de métiers nouveaux ou plus anciens. Les métaux touchent à tous les non ferreux, dont beaucoup sont indispensables à la vie domestique et à la construction, jusqu'à l'invention de la période : le zinc. Il en va un peu de même du fer : il est surtout de seconde fusion et utilisé pour la transformation en petits objets de quincaillerie. Les traits communs à cette métallurgie parisienne sont une place essentielle tenue par la récupération et des ateliers modestes. Les grandes entreprises parisiennes liées à la mécanique lourde seront tardives et généralement animées par des Britanniques. S'il faut chercher une spécificité métallique à Paris, c'est plutôt dans l'orfèvrerie et l'utilisation des métaux précieux! La capitale s'y taille une réputation internationale, jusqu'à concurrencer Birmingham dans le domaine du bouton métallique, pourtant place forte s'il en est de la production industrielle insulaire. La mécanique parisienne est plutôt de petite taille et de précision: horlogerie et métrologie sont de réputation internationale durant cette période.

7 Dans une dernière partie, Les arts industriels, André Guillerme s'attache aux nouveaux métiers et à l'air du temps. C'est là qu'il nous parle de l'amour du blanc et de la lumière des Parisiens, sans déroger à sa méthode générale d'étude. Cela se décline en peindre la ville dont les fonds blancs à la céruse sont aussi dangereux que le mercure des chapeliers, sans en empêcher le succès commercial éclatant. C'est ensuite le blanc du linge allant $\mathrm{du}$ "bouillir » à cette invention bien datée et bien française de l'eau de javel, et à l'apparition précoce de la blanchisserie industrielle dans la capitale. Enfin, 
l'auteur présente une compilation de travaux déjà nombreux mais parfaitement mis en perspective sur l'incroyable dynamisme d'innovations dans le domaine de l'éclairage durant cette période. Paris est devenue « ville lumière » à ce moment-là.

Il faut souligner la très haute qualité générale de l'ouvrage qui ne se contente pas de décrire et de compiler, ce qui est déjà beaucoup, ni même de mettre en perspective statistique et de souligner les dynamiques professionnelles et sociales à l'œuvre. André Guillerme, tout au long de son propos, ne cesse de suivre et d'alimenter deux autres grandes problématiques qui lui tiennent à cœur et dont il est aujourd'hui l'un des meilleurs spécialistes : la géographie urbaine de l'industrie et l'analyse de ses mutations en premier lieu, les pollutions de l'industrie et leurs conséquences sociales et structurelles en second lieu. Il faut au final saluer un travail brillant, parfaitement conduit et d'une très grande richesse, qui marque une étape nouvelle dans notre compréhension des processus d'industrialisation en Europe.

\section{AUTEURS}

\section{MICHEL COTTE}

Centre François Viète, Université de Nantes 